\title{
IL-17A brings new recruits to EAE
}

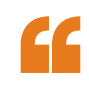

IL-17A pro-
duced by
antigen-
nonspecific
$\gamma \delta$ T cells in
the periphery
recruits innate
immune cells
that prime
pathogenic
T cells

T cells

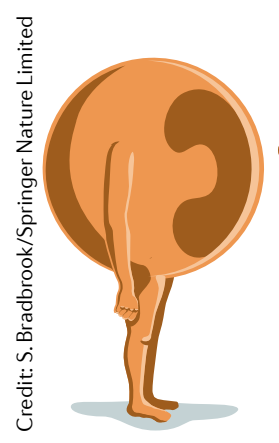

IL-17A and IL-17A-producing $\mathrm{T}$ cells are key mediators of various autoimmune diseases, including multiple sclerosis. Generally, IL-17A produced by autoantigen-specific $\mathrm{CD}^{+} \mathrm{T}$ cells in the diseased tissue is thought to promote the production of inflammatory mediators by epithelial cells. However, new findings published in Immunity suggest that, in a mouse model of $\mathrm{T}$ cell-driven central nervous system (CNS) inflammation, IL-17A produced by antigen-nonspecific $\gamma \delta$ T cells in the periphery recruits innate immune cells that prime pathogenic T cells.

First, McGinley et al. showed that, as expected, $I l 17 a^{-/-}$mice are resistant to the induction of myelin oligodendrocyte glycoprotein (MOG)-induced experimental autoimmune encephalomyelitis (EAE), which correlated with a reduced number of pathogenic $\mathrm{T}$ cells in the CNS. However, treatment of mice with a neutralizing antibody to IL-17A during the effector phase of EAE did not affect disease severity. By contrast, antibody treatment during the induction phase delayed the onset of EAE and attenuated symptoms, which was associated with reduced numbers of pathogenic T cells in the spleen. Thus, IL-17A seems to have an early role in the peripheral activation of T cells that later infiltrate the CNS. Indeed, the frequencies of cytokineproducing $\mathrm{CD}^{+}$and $\gamma \delta$ T cells were significantly reduced in the lymph nodes of $I l 17 a^{-/-}$mice compared with wild-type mice immunized with MOG plus adjuvant, and the $I l 17 a^{-/-}$ $\mathrm{CD}^{+}{ }^{+} \mathrm{T}$ cells had reduced expression of the integrin VLA4, which drives $\mathrm{T}$ cell infiltration of the CNS.

In a transfer model, donor T cells from MOG-immunized wild-type or $I l 17 a^{-/-}$mice were expanded in vitro, with MOG alone or with MOG plus IL- $1 \beta$ and IL-23, then transferred into naive wild-type mice. Both wild-type and $I l 17 a^{-1-}$ T cells induced severe EAE in recipient mice after culture with MOG, IL-1 $\beta$ and IL-23, which supports the hypothesis that IL-17A production by $\mathrm{T}$ cells is not required for the effector phase of disease, provided that polarizing cytokines such as IL-1 $\beta$ are provided during the induction phase. The authors identified $\mathrm{V} \gamma 4^{+} \gamma \delta \mathrm{T}$ cells, which can be activated by IL- $1 \beta$ and IL-23 independently of antigen, as an important cellular source of IL-17A during this induction phase of EAE.

In keeping with the inductive role of IL- $1 \beta$ and IL-23 shown in the transfer model, the authors proposed that IL-17A might be required for early production of IL- $1 \beta$ and/or IL-23. They found significantly reduced expression of $I l 1 b$ in the lymph nodes of $I l 17 a^{-/-}$mice, and administration of IL- $1 \beta$ to $I l 17 a^{-/-}$ mice during EAE induction restored disease susceptibility.
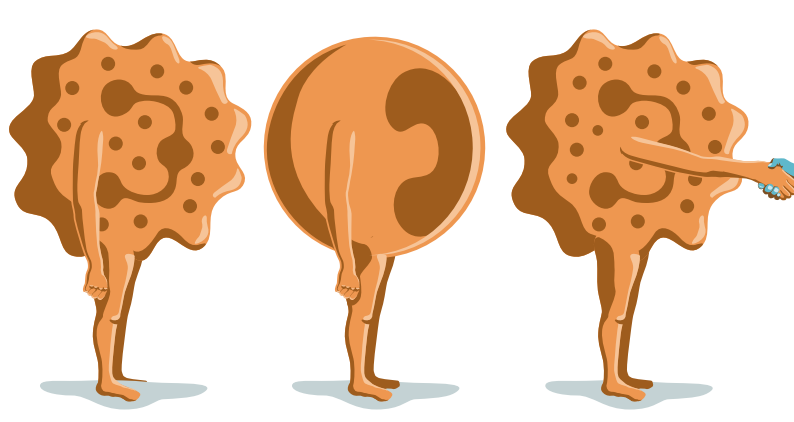

Furthermore, treatment with antibody to IL- $1 \beta$ during EAE induction in wild-type mice reduced the severity of disease, which was associated with reduced activation of MOG-specific T cells in the lymph nodes. Together, the results suggest that the role of IL-17A in disease induction involves promoting IL- $1 \beta$ production to activate encephalitogenic T cells.

In looking for the cellular source of this IL-17A-induced IL- $1 \beta$ production, they found high levels of expression of IL-17RA on neutrophils and Ly6 $\mathrm{C}^{\text {hi }}$ inflammatory monocytes early in EAE. $I l 17 a^{-/-}$mice had reduced numbers of neutrophils and Ly6 $\mathrm{C}^{\mathrm{hi}}$ monocytes in the spleen after EAE induction. However, genetic ablation of IL-17RA in myeloid cells did not render mice resistant to EAE induction, which suggests that IL-17A acts on non-myeloid cells to recruit the myeloid cells that produce IL-1 $\beta$. Further experiments completed the missing details by showing that epithelial cells produce chemokines such as CXCL1 and CXCL2 in response to IL-17A, which in turn recruit IL- $1 \beta$-producing neutrophils and monocytes.

Thus, the results suggest a positive-feedback loop during EAE induction whereby IL-17A production, mainly by $\gamma \delta$ T cells, indirectly induces the recruitment of myeloid cells that produce IL- $1 \beta$, which in turn can synergise with IL-23 to activate $\gamma \delta$ T cells and later $\mathrm{CD} 4^{+} \mathrm{T}$ cells that mediate the effector phase of disease.

Kirsty Minton

ORIGINAL ARTICLE McGinley, A. M. et al. Interleukin-17A serves a priming role in autoimmunity by recruiting IL-1 $\beta$-producing myeloid cells that promote pathogenic T cells. Immunity https://doi.org/10.1016/j.immuni. 2020.01.002 (2020) 